\title{
Damage Identification of a Derrick Steel Structure Based on the HHT Marginal Spectrum Amplitude Curvature Difference
}

\author{
Dongying Han, ${ }^{1}$ Shimin Wei, ${ }^{1}$ Peiming Shi, ${ }^{2}$ Ying Zhang, ${ }^{3}$ Kai Gao, ${ }^{1}$ and Nengyuan Tian ${ }^{1}$ \\ ${ }^{1}$ School of Vehicles and Energy, Yanshan University, Qinhuangdao 066004, China \\ ${ }^{2}$ School of Electrical Engineering, Yanshan University, Qinhuangdao 066004, China \\ ${ }^{3}$ School of Electrical and Computer Engineering, Georgia Institute of Technology, Atlanta, GA 30332, USA
}

Correspondence should be addressed to Dongying Han; dongying.han@163.com

Received 25 April 2017; Revised 18 July 2017; Accepted 7 August 2017; Published 17 September 2017

Academic Editor: Giada Gasparini

Copyright (C) 2017 Dongying Han et al. This is an open access article distributed under the Creative Commons Attribution License, which permits unrestricted use, distribution, and reproduction in any medium, provided the original work is properly cited.

\begin{abstract}
For the damage identification of derrick steel structures, traditional methods often require high-order vibration information of structures to identify damage accurately. However, the high-order vibration information of structures is difficult to acquire. Based on the technology of signal feature extraction, only using the low-order vibration information, taking the right front leg as an example, we analyzed the selection of HHT marginal spectrum amplitude and the calculation process of its curvature in practical application, designed the damage conditions of a derrick steel structure, used the index and intrinsic mode function (IMF) instantaneous energy curvature method to perform the damage simulation calculation and comparison, and verified the effect of identifying the damage location in a noisy environment. The results show that the index can accurately determine the location of the damage element and weak damage element and can be used to qualitatively analyze the damage degree of the element; under the impact load, the noise hardly affects the identification of the damage location. Finally, this method was applied to the ZJ70 derrick steel structure laboratory model and compared with the IMF instantaneous energy curvature method. We verified the feasibility of this method in the damage location simulation experiment.
\end{abstract}

\section{Introduction}

Derrick steel structures play an important role in the oil and gas exploration and development $[1,2]$. During long-term service, because of various factors such as disassembly and corrosion, damage inevitably occurs, which gradually reduces the safety performance and carrying capacity of the derrick steel structures and forms security risks [3-5]. In recent years, the damage detection method based on structural signal feature extraction $[6,7]$ has attracted the attention of many scholars; this method mainly uses the vibration signal from the vibration sensors to collect structural damage. Using signal-processing methods, the appropriate damagesensitive indicators are analyzed, and further structuraldamage identification or health monitoring is realized [8].

Huang et al. proposed Hilbert-Huang transform (HHT) method, which is a new, self-adaptive frequency analysis method [9], and included the empirical mode decomposition (EMD) and Hilbert transform (HT); the core is the
EMD. Chen et al. used the instantaneous frequency of IMFs (intrinsic mode functions) as the component damage index of structure damage detection [10-12]. Pines and Salvino combined the EMD with Hilbert transform to obtain the phase of the component signals and used the phase information of different degrees of freedom to identify the structure damage [13]. Li et al. [14] used the method of wavelet analysis to analyze the maximum energy of the intrinsic mode functions (IMFs) component, which includes the wavelet coefficients for damage identification. Cheraghi and Taheri [15] analyzed the energy of the IMF component signal, selected the effective characteristic information as the damage sensitivity index, and applied it to identify the pipeline structure damage. Rezaei and Taheri $[16,17]$ used the energy of the first-order IMF component after the decomposition of the signal EMD as a damage sensitivity index to diagnose the damage of the pipeline structure. Chen et al. proposed to show the status of damage material wing box of the material of the feature vector-relative variation of the instantaneous frequency [18]. 
Cao et al. [19] proposed a structural-damage early-warning method based on the EMD, where the structure before and after damage in the component of the IMF's energy distribution changes as the damage-sensitive index, and applied the method to model Health Monitoring Benchmark structure damage identification. Ren et al. [20] applied the improved HHT method to the damage identification of engineering structures and proposed the method to identify the damage location of the structure using the structural before and after the damage in the first-order response to a first-order IMF feature energy ratio. Wang et al. applied the method to the bridge structure. The HHT theory has not been used in complex structures, such as derrick steel structures, but it has been used in simple structures in the literature [21]. Li et al. applied the method to the crack identification of the rotor [22]. This paper realized the damage identification of the derrick steel structure based on the HHT marginal spectrum amplitude curvature difference and only used a low-level vibration information structure. The simulation calculated the damage identification of the derrick and compared it with the IMF instantaneous energy curvature difference. Finally, the feasibility of the method was verified with derrick damage location simulation experiments.

\section{HHT Marginal Spectrum Amplitude Curvature}

2.1. HHT Theory. The HHT method consists of two parts: EMD and HT. The original signal $x(t)$ is decomposed into a series of IMFs and a residual function by EMD:

$$
x(t)=\sum_{i=1}^{n} c_{i}+r_{n}
$$

Hilbert transformation of the IMF component:

$$
\widehat{c}_{i}=\frac{1}{\pi} \int_{-\infty}^{+\infty} \frac{c_{i}(\tau)}{t-\tau} d \tau
$$

Based on this function, the analytic signal is established, and the instantaneous amplitude and phase function are obtained:

$$
\begin{aligned}
z_{i} & =c_{i}+j \widehat{c}_{i}=a_{i} e^{j \varphi_{i}}, \\
a_{i} & =\sqrt{c_{i}^{2}+\widehat{c}_{i}^{2}}, \\
\varphi_{i} & =\arctan \frac{\widehat{c}_{i}}{c_{i}} .
\end{aligned}
$$

The instantaneous frequency is defined as the derivative of the instantaneous phase:

$$
\omega_{i}=\frac{d \varphi_{i}}{d t}
$$

Signal $x(t)$ can be expressed as

$$
x(t)=\operatorname{RE} \sum_{i=1}^{n} a_{i} e^{j \varphi_{i}}=\operatorname{RE} \sum_{i=1}^{n} a_{i} e^{j \int \omega_{i} d t} .
$$

Here, we ignore the residual component of the original signal $r_{n}$; RE denotes the real part. We call the above formula on the right part of the equal sign the Hilbert spectrum:

$$
H(\omega, t)=\operatorname{RE} \sum_{i=1}^{n} a_{i}(t) e^{j \int \omega_{i}(t) d t} .
$$

Hilbert marginal spectrum is obtained by integrating the above formula with time:

$$
h(\omega)=\int_{0}^{T} H(\omega, t) d t .
$$

2.2. HHT Marginal Spectrum Amplitude Curvature. After the structure is applied to the vibrational excitation, the vibration response signal of different parts of the structure is extracted. After the signal is decomposed by the EMD, a series of IMF components is obtained, the main IMF component is selected, and the marginal spectrum amplitude is calculated. Then, the relative HHT marginal spectrum amplitude at different parts of the structure is

$$
R_{i}=\frac{F_{i}}{F_{0}}
$$

where $F_{i}$ is the HHT marginal spectrum of different parts of the structure and $F_{0}$ is the HHT marginal spectrum of the structure reference position.

The HHT marginal spectrum amplitude curvature of different parts of structures is approximately calculated by the central difference method:

$$
\Phi_{i}=\frac{R_{i+1}-2 R_{i}+R_{i-1}}{l^{2}}
$$

where $R_{i}$ is calculated at the site of the relative HHT marginal spectrum amplitude, $R_{i+1}$ and $R_{i-1}$ are calculated at the sites adjacent to the relative HHT marginal spectrum amplitude, and $l$ is the distance between adjacent parts.

The difference in HHT marginal spectrum amplitude curvature is

$$
\Delta \Phi_{i}=\Phi_{u i}-\Phi_{d i}
$$

where $\Phi_{u i}$ is the HHT marginal spectrum amplitude curvature before structural damage.

\section{Extracting the Signal Characteristics of the Derrick Steel Structure}

3.1. Establishment of the Simulation Model of the Derrick Steel Structure. The model of the derrick steel structure is $2.951 \mathrm{~m}$ high, and the maximum hook load is $13.9 \mathrm{kN}$. Its material is Q235 steel. The structure was divided into 274 elements and 142 nodes, and its finite-element model is shown in Figure 1. The right front pillar nodes of the derrick steel structure were numbered 1-20 from the bottom. There was one element between every two nodes, and the elements were numbered 1-19 from the bottom to the top. 


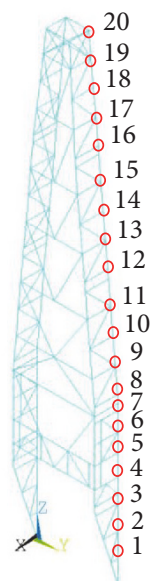

FIGURE 1: Finite-element model of the derrick steel structure.

3.2. Derrick Steel Structure Model Analysis. The modal analysis of the model shows that the first three orders of the frequency were 17.53, 18.0, and $38.71 \mathrm{~Hz}$. The first-order vibration mode was mainly the left-and-right first-order bending overall vibration; the second-order vibration mode was mainly the front-and-back first-order bending overall vibration; the third-order vibration mode was mainly torsional vibration.

We used a random-noise acceleration load in the vibration model of the derrick steel structure; the sampling frequency of the random-noise acceleration loads was $1000 \mathrm{~Hz}$; the amplitude was $1.2 \mathrm{~m} / \mathrm{s}^{2}$; and the load acted on node 20 along the direction of $y$-axis for $2 \mathrm{~s}$. We used the complete method to solve the transient dynamics, and the acceleration response of nodes 1-20 of the right front pillar of the derrick steel structure in the $y$ direction was extracted.

3.3. Extracting the HHT Marginal Spectrum Amplitude Curvature. Our example structure has 15 nodes. The acceleration response of the Fourier spectral analysis in Figure 2 shows that random loads can stimulate the first- and third-order frequencies $(17.53 \mathrm{~Hz}$ and $38.71 \mathrm{~Hz})$ in the derrick steel structure, and the first-order vibration is prioritized.

Considering the complexity of the derrick steel structure, the nodes in the lower part of the derrick steel structure cannot stimulate the third-order frequency under a random load. Therefore, we used the bandpass filter and HHT combination of methods to extract the first-order vibration information of the derrick steel structure and determine the cut-off frequency of $17 \mathrm{~Hz}$ and $18 \mathrm{~Hz}$. We filtered the other frequency components using the EMD decomposition, as shown in Figure 3.

Figure 3 shows that the main characteristic information of the signal is concentrated in the first-order IMF component; therefore, we only extracted the marginal spectrum amplitude of the first-order IMF component. The marginal spectrum of the first-order IMF component of node 15 is shown in Figure 4. We could extract the marginal spectrum amplitude of the derrick steel structure from its vibration in
TABLE 1: Damage condition of the derrick steel structure.

\begin{tabular}{lccc}
\hline Condition & Damage degree & Damage type & Damage position \\
\hline 1 & $5 \%$ & & 16 \\
2 & $20 \%$ & Single damage & 10 \\
3 & $30 \%$ & & 5 \\
4 & $5 \%$ & & 8,14 \\
5 & $10 \%$ & Double damage & 5,13 \\
6 & $5 \%, 10 \%$ & & 5,13 \\
\hline
\end{tabular}

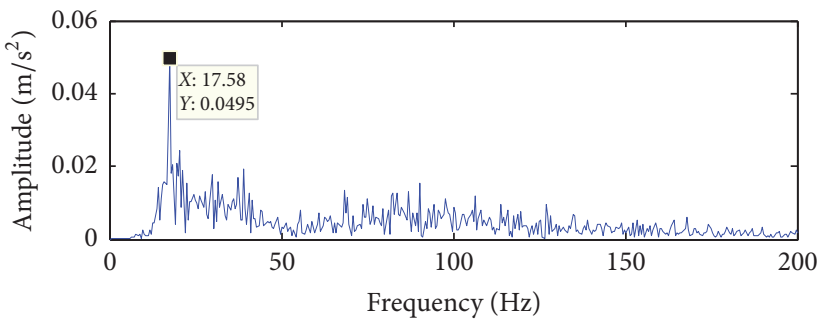

FIGURE 2: Spectral analysis of the acceleration response of node 15 in the derrick steel structure.

the first-order frequency. In the derrick, we selected node 1 as the reference point and calculated the HHT marginal spectrum amplitude curvature of nodes 1 20.

\section{Simulation Analysis}

4.1. Damage Condition Design. In the practical condition of the derrick steel structure, damage most likely occurs in the front pillar, so we selected the right front pillar of the derrick steel structure for the study. Damage was set up in two cases: single damage and double damage. Damage can be achieved by reducing the stiffness of the element, and the specific damage conditions are shown in Table 1.

4.2. Identifying Damage Location. The nonstationary signal can be decomposed into an intrinsic mode function (IMF) and a residual function by empirical modal decomposition. Each component of the IMF contains the local characteristics of different time scales in the original signal. The IMF instantaneous energy reflects the change over time of each component. To verify the effectiveness of this method, we used the HHT marginal spectrum amplitude curvature difference and IMF instantaneous energy curvature difference to identify the damage location under six damage conditions. We calculated the derrick steel structure in good conditions and six damage conditions of the HHT marginal spectrum amplitude curvature and IMF instantaneous energy curvature of nodes 1 20. Then, we compared our results with the corresponding node of curvature difference before and after the derrick steel structure was damaged. The sensor is disturbed by external noise in practical conditions, so we verified the effect of identifying the damage location in a noisy environment.

The above analysis is shown in Figure 5. Figures 5(a)-5(d) show the single-damage conditions, where elements 16,10 , 

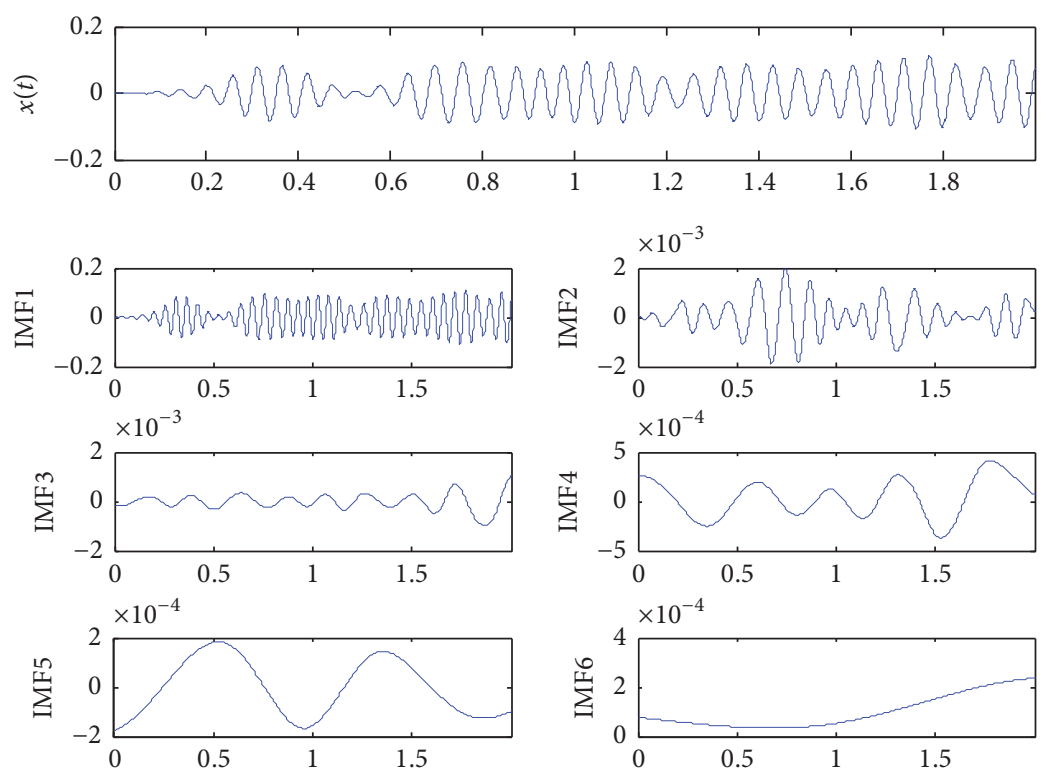

FIGURE 3: First-order vibration response of node 15 in the derrick steel structure and EMD.

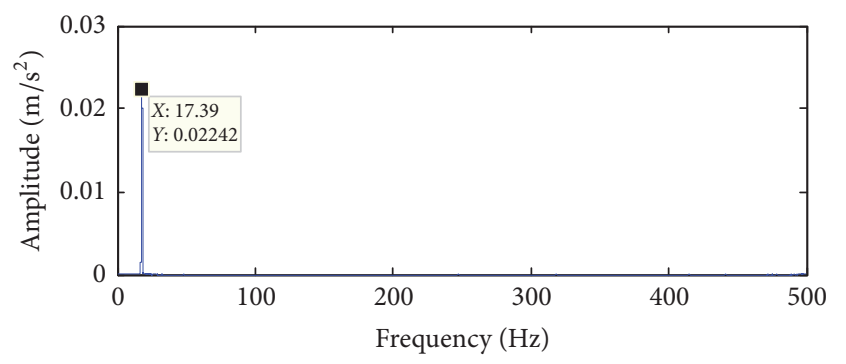

FIGURE 4: First-order IMF component marginal spectrum of node 15 in the derrick steel structure.

and 5 were clearly damaged. Figures $5(\mathrm{a})$ and $5(\mathrm{~d})$ show the $5 \%$ damage condition of element 16 . Figure $5(\mathrm{e})$ is the relation curve between the HHT marginal spectrum curvature difference and the node in $10 \%$ random noise of damaged element 5; Figures 5(f)-5(i) show the doubledamage conditions. We can clearly identify the position of the upper and lower parts of the damaged element in the pillar. Figures $5(\mathrm{~g})$ and $5(\mathrm{~h})$ compare the same damage location with different degrees of damage, and the upper part of the damage element in the pillar of Figure 5(h) is easier to identify than that in Figure 5(g). Figure 5(j) is the relation curve between the HHT marginal spectrum curvature difference and the node in $10 \%$ random noise of damaged elements 5 and 13. The comparison shows that the noise under the impact load hardly affected the damage location identification of the derrick steel structure. Therefore, both methods can identify the damage location of the derrick, and the rule based on the HHT method is as follows. The HHT marginal spectrum amplitude curvature difference of the damaged element at two ends of the node mutates, and neighboring nodes have opposite signs. The absolute value of one curvature difference of the node is the maximum of nearby nodes, which can make the HHT marginal spectrum curvature difference of adjacent nodes increase. For the double-damaged places, the HHT marginal spectrum amplitude curvature difference is sensitive to the lower part of the damaged element of the pillar identification. When the damaged position is identical, with the increase in damage degree, it is easier to identify the position of the upper damaged element, and the position of the weakly damaged element can be correctly identified.

4.3. Damage Degree Identification. Supposing that the stiffness of element 13 of the right front pillar of the derrick steel structure successively was reduced by $5 \%, 10 \%, 15 \%, 20 \%$, $25 \%, 30 \%, 35 \%, 40 \%, 45 \%$, and $50 \%$, we calculated the HHT marginal spectrum amplitude curvature difference of node 13 of element 13 at the corresponding damage degree. Figure 6 shows the histogram of the relationship between the absolute value of the HHT marginal spectrum amplitude curvature difference and the damage degree.

Figure 6 shows that the absolute value of the HHT marginal spectrum amplitude curvature difference of node 13 increases with the increase in damage degree. Therefore, we can qualitatively analyze the damage degree of a derrick steel structure based on the HHT marginal spectrum amplitude curvature difference.

\section{Experimental Analysis}

5.1. Damage Location Simulation and Sensor Layout. The ZJ70-type derrick steel structure laboratory model was made according to the prototype equipment of a $7000 \mathrm{~m}$ drilling rig with a proportion of $1: 18$. The model is a front-opening model with beam pillars and is divided into four segments. The upper part is the overall structure of the closed segment; the other three segments have a front-opening shape of " $\cap$." The components of the main body are connected with pins, and its physical model is shown in Figure 7. 


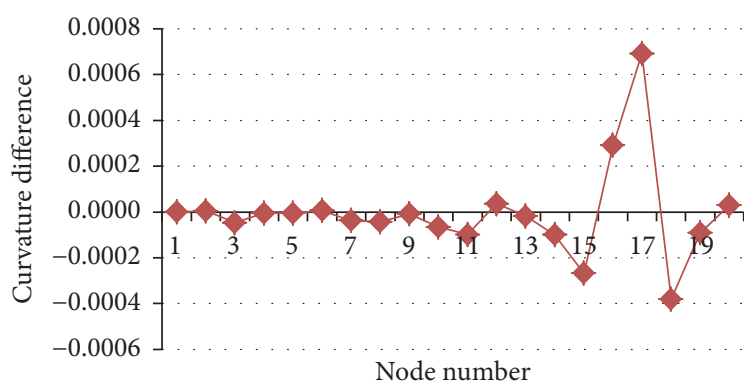

(a) Condition 1 (damage of unit 16; HHT method)

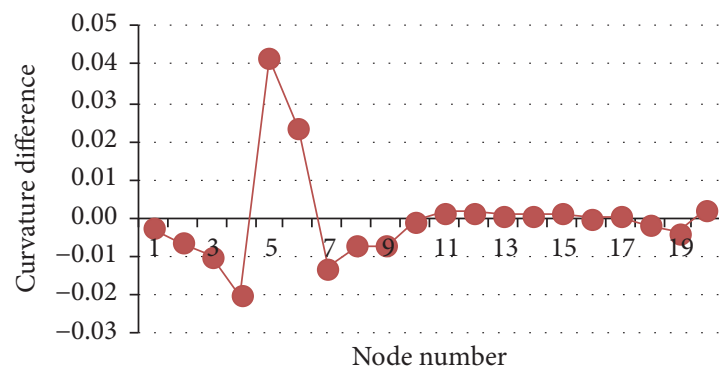

(c) Condition 3 (damage of unit 5; HHT method)

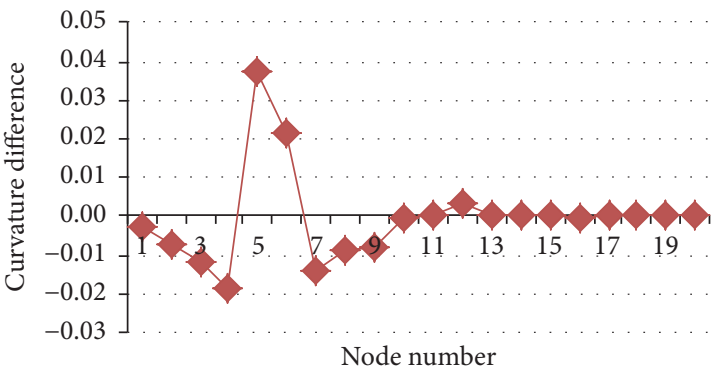

(e) $10 \%$ random noise (damage of unit 5 )

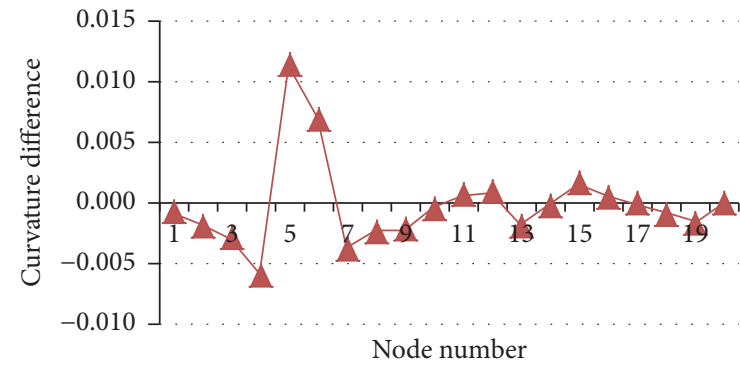

(g) Condition 5 (damage of units 5 and 13; HHT method)

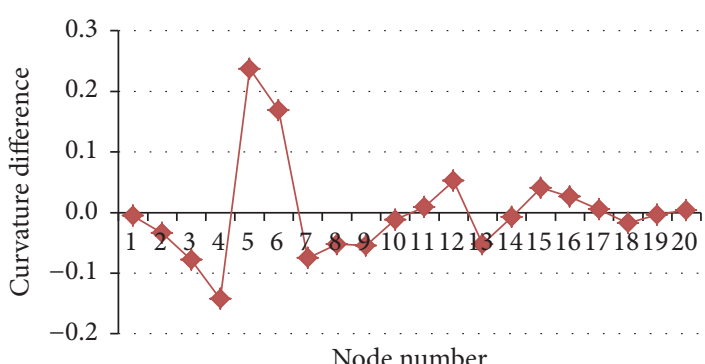

Node number

(i) Condition 6 (damage of units 5 and 13; IMF method)

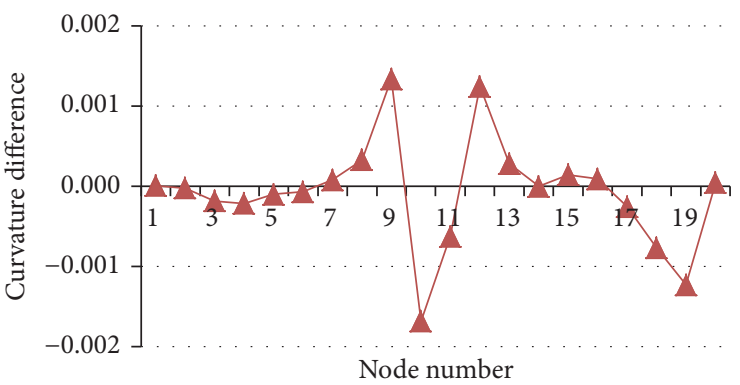

(b) Condition 2 (damage of unit 10; HHT method)

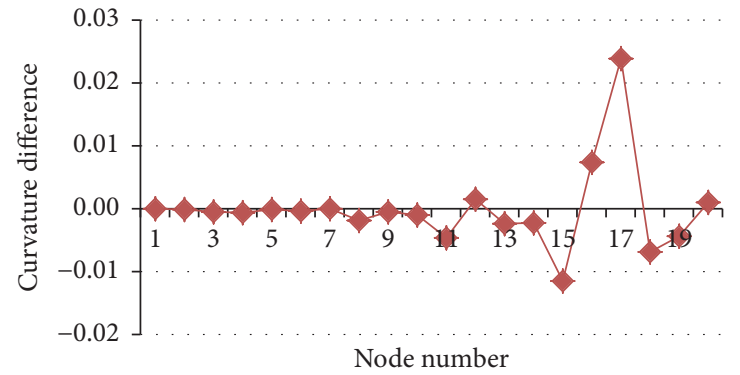

(d) Condition 1 (damage of unit 16; IMF method)

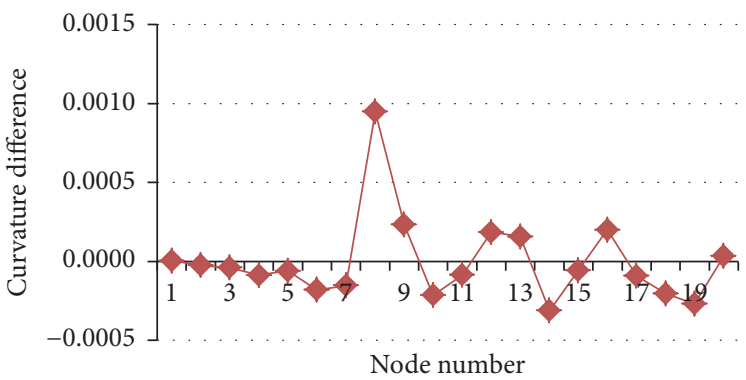

(f) Condition 4 (damage of units 8 and 14; HHT method)

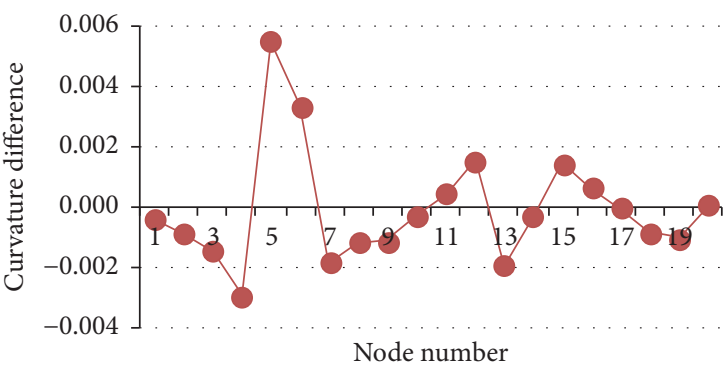

(h) Condition 6 (damage of units 5 and 13; HHT method)

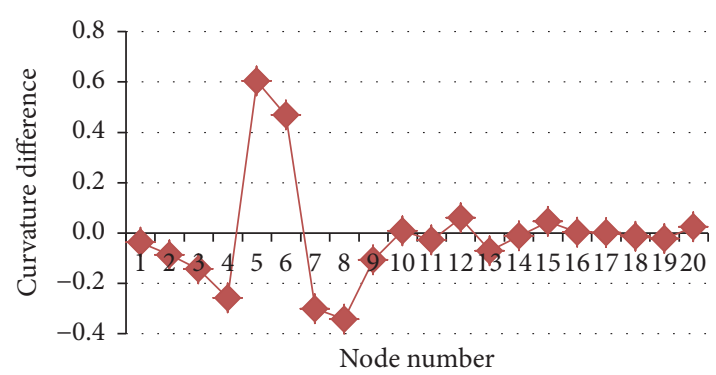

(j) 10\% random noise (damage of units 5 and 13)

FIGURE 5: Relationship between the difference of the HHT marginal spectrum curvature and the nodes. 
TABLE 2: Damage experimental conditions of the derrick steel structure.

\begin{tabular}{lccc}
\hline Condition & Damage form & Damage type & Damage position \\
\hline 1 & & Single damage & The place between second segment and third segment \\
2 & Pin loose & Single damage & The place between third segment and forth segment \\
3 & & Double damage & Integrating the first two kinds of damage location \\
\hline
\end{tabular}

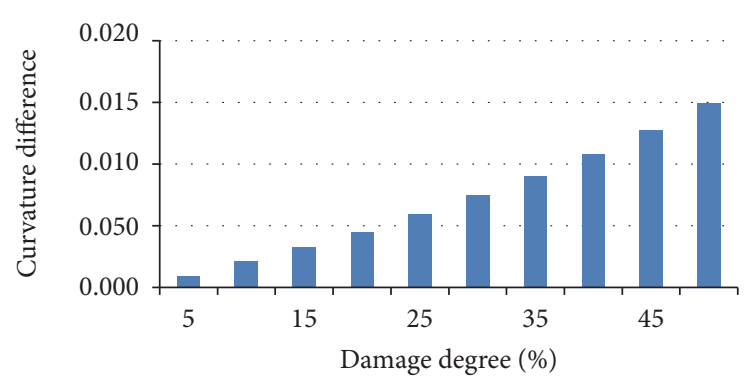

FIGURE 6: Relationship between the difference of the HHT marginal spectrum curvature and the damage degree.

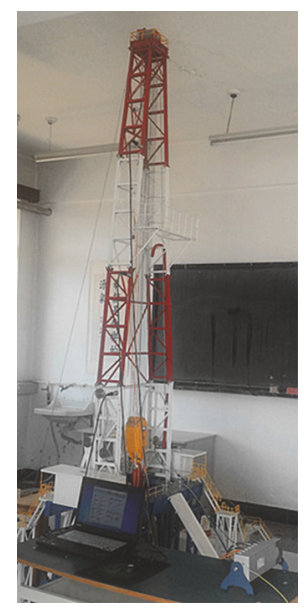

FIGURE 7: Laboratory model of the ZJ70-type derrick steel structure.

We numbered the segments of the derrick steel structure number as 1-4 from top to bottom. Because of the limited conditions, the experiment of the derrick steel structure was based on one of the two front-opening pillars as the object of study, and the damage forms are simulated by loosening the pin between two segments. We simulated 3 damage conditions. The specific damage conditions are shown in Table 2.

In the experiment, 12 acceleration sensors were used to collect the vibration signals of different parts of the derrick steel structure. The sensors were numbered 1-12 from top to bottom. Conditions 1 and 2 simulated the damage between sensors 6 and 7; Condition 3 simulates the damage between sensors 3 and 4 and the damage between sensors 9 and 10. Figure 8 shows the measured distance between adjacent acceleration sensors under three damage conditions. When we calculated the HHT marginal spectrum amplitude curvature, we selected sensor 1 as the reference point.

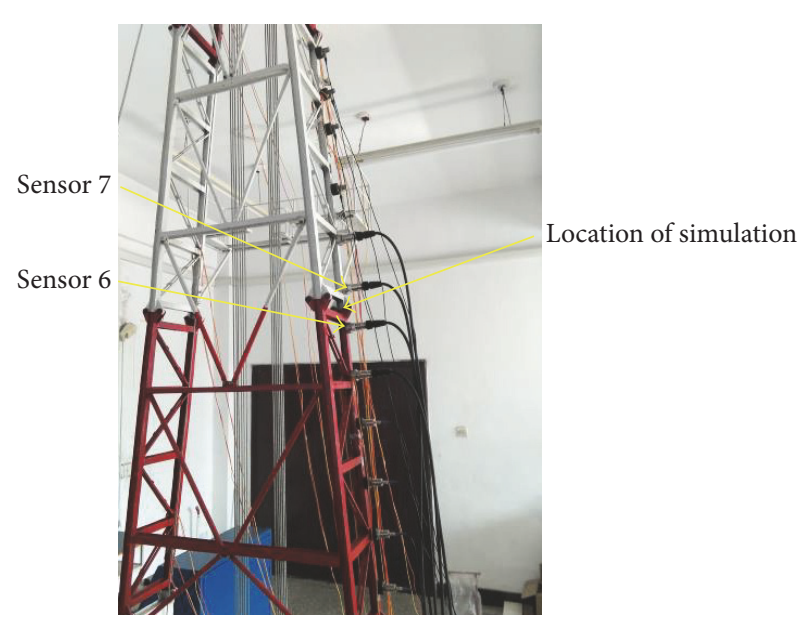

(a) Single damage

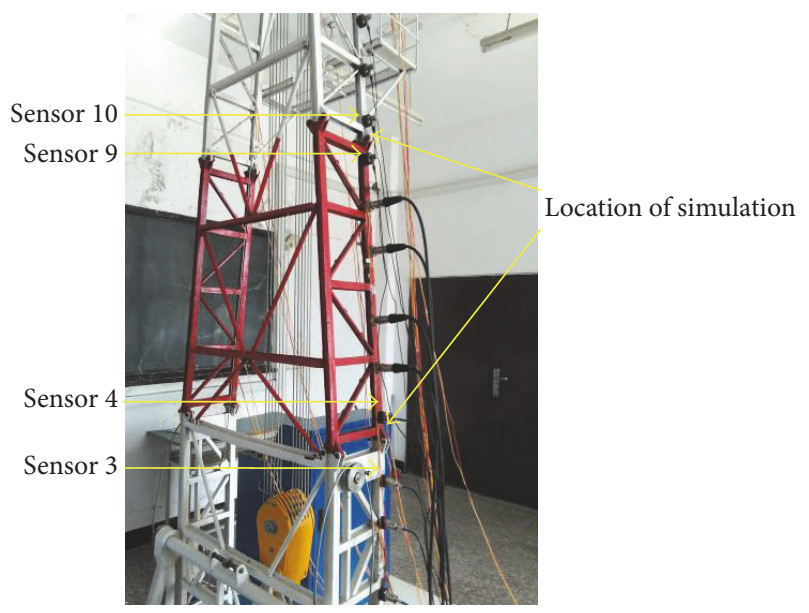

(b) Double damage

FIGURE 8: Damage location simulation and sensor layout.

5.2. Experimental Signal Acquisition. The vibration excitation mode in the experiments was marked to ensure that the vibration position was identical before and after the simulation of the derrick steel structure. The 12 sensors simultaneously collected the vibration signal of the derrick steel structure; the interface shows the time-domain signal window in real time and 12 channels. To easily observe the signal collected by each sensor, Figure 9 is based on Condition 1 as an example, and we intercepted the time-domain signal of sensors 1-12 of the derrick steel structure before it became damaged.

5.3. Analysis of Experimental Results. We calculated the HHT marginal spectrum amplitude curvature difference and IMF instantaneous energy curvature difference of the derrick steel 

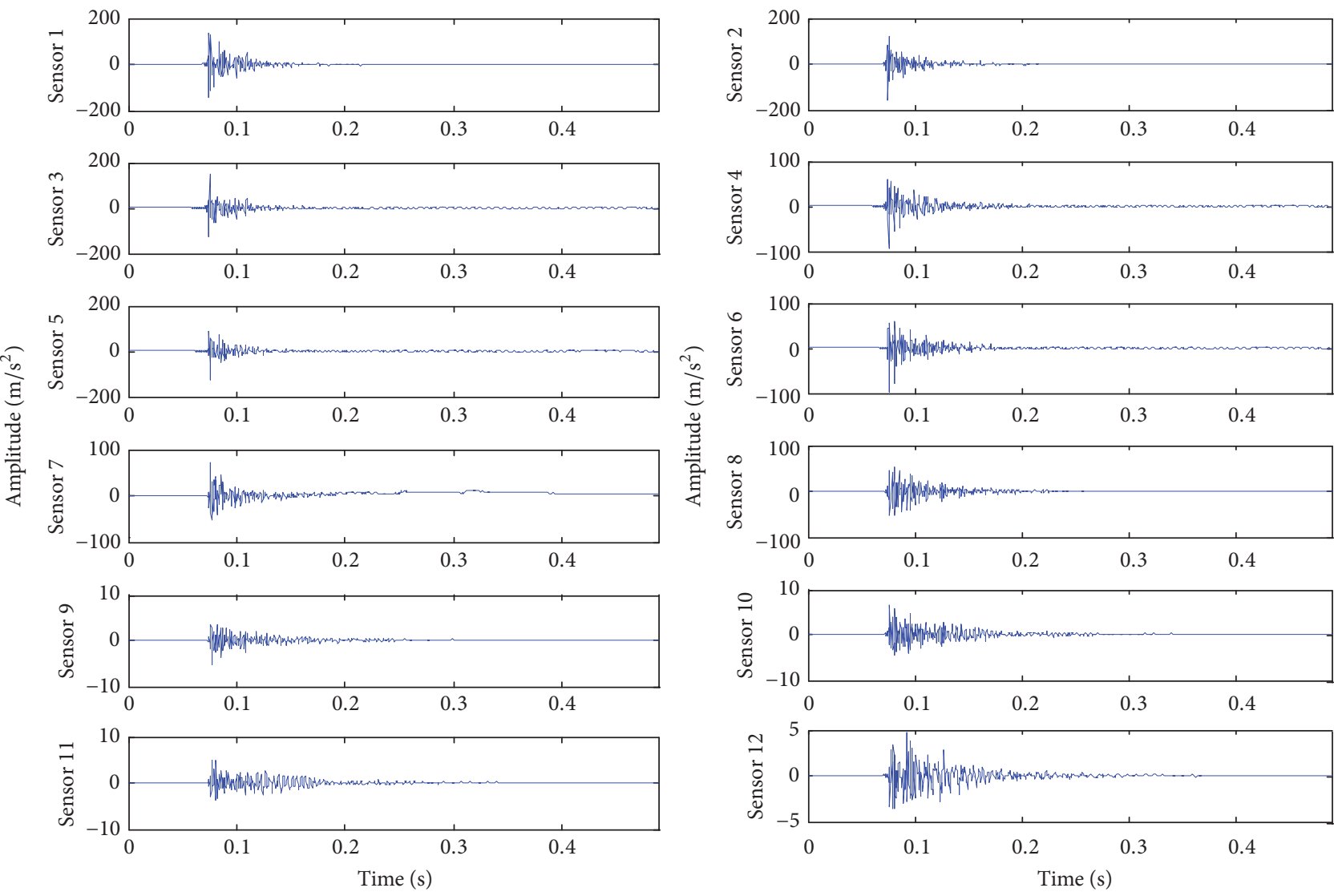

(a) Before being damaged
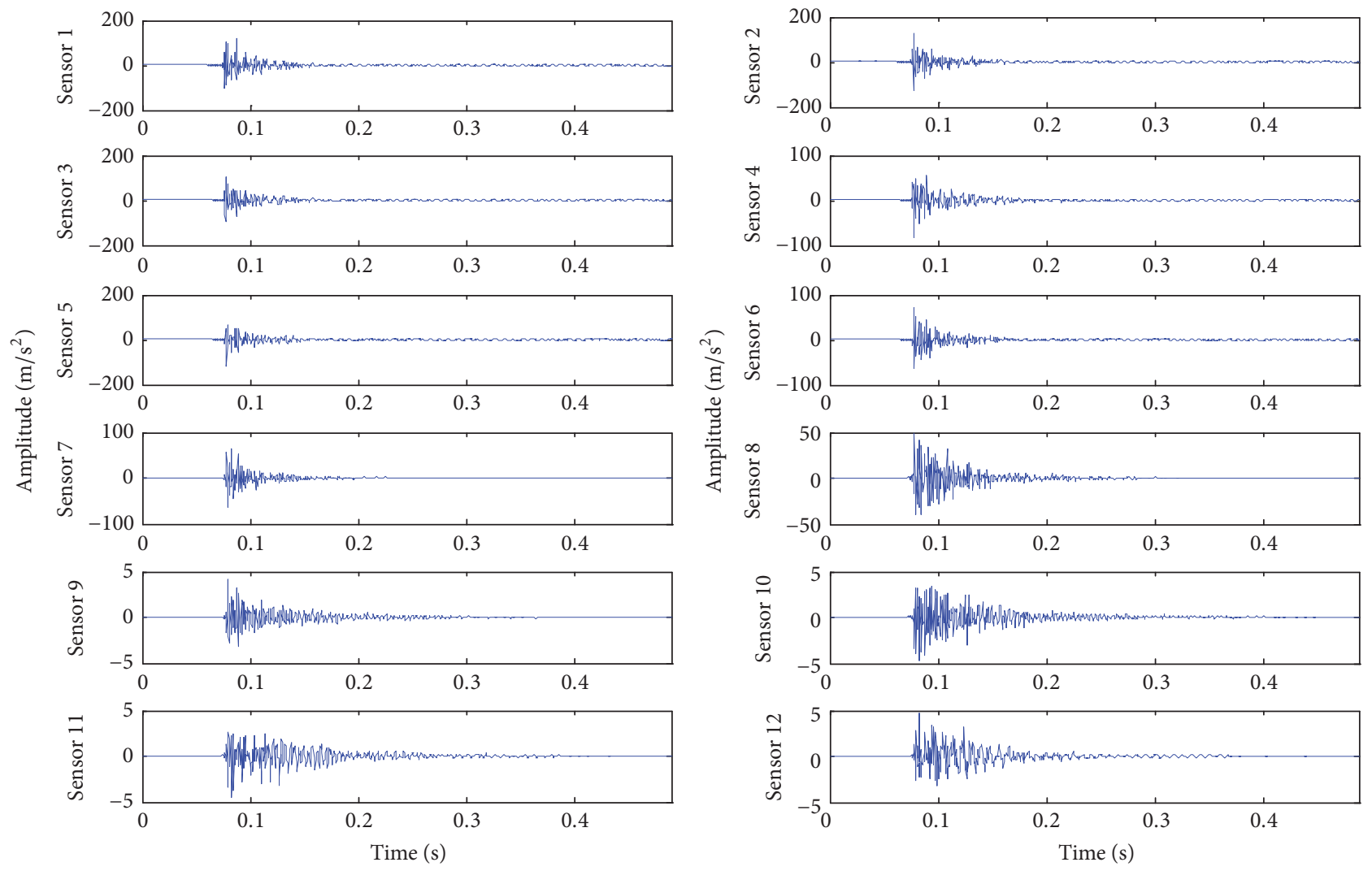

(b) After being damaged

FIgURE 9: Time-domain signal of sensors 1-12 before and after the damage of the derrick steel structure under Condition 1. 


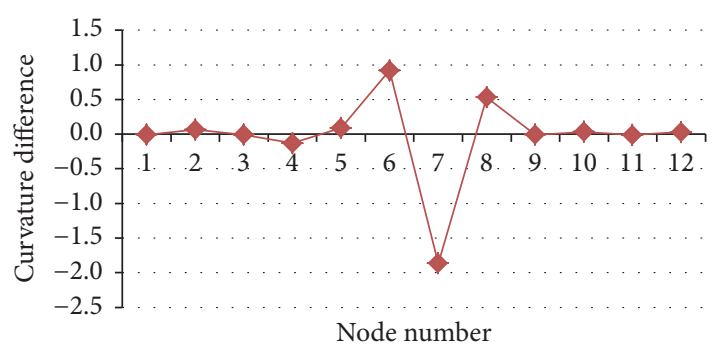

(a) Condition 1 (Sensors 6 and 7; HHT method)

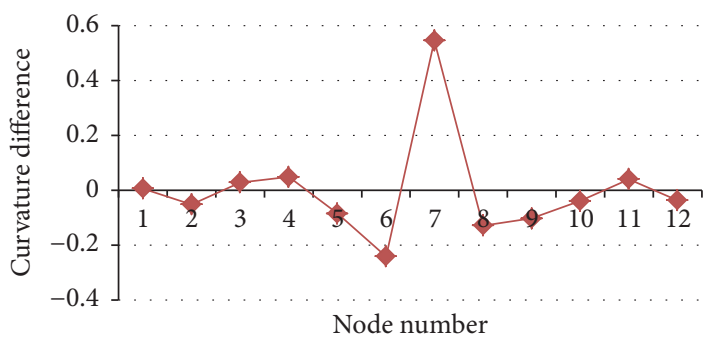

(c) Condition 2 (Sensors 6 and 7; HHT method)

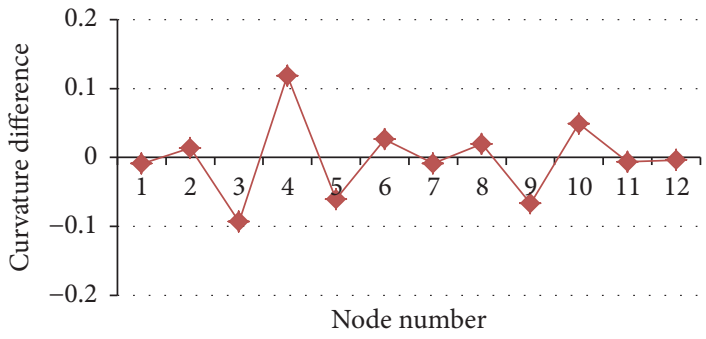

(e) Condition 3 (Sensors 3, 4, 9, and 10; HHT method)

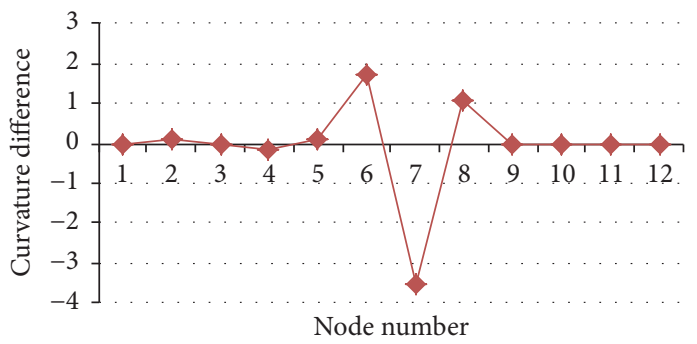

(b) Condition 1 (Sensors 6 and 7; IMF method)

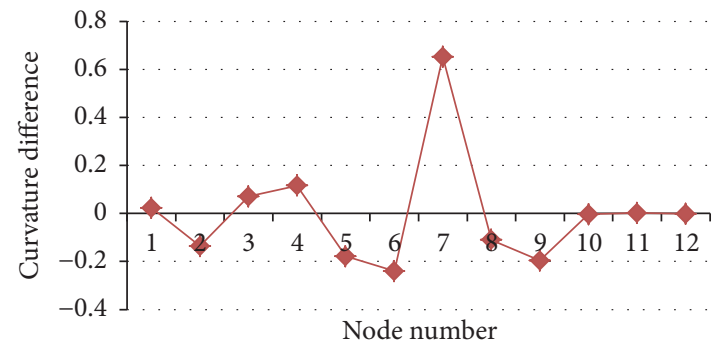

(d) Condition 2 (Sensors 6 and 7; IMF method)

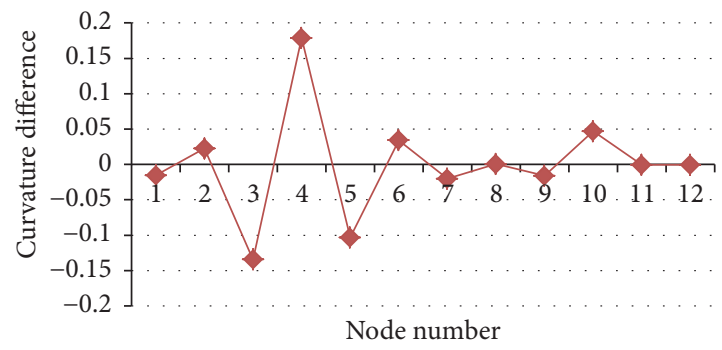

(f) Condition 3 (Sensors 3, 4, 9, and 10; IMF method)

FIGURE 10: Relationship between the HHT marginal spectrum curvature difference or IMF instantaneous energy curvature difference and the sensors.

structure in three conditions and show its relationship with sensors 1-12 in Figure 10.

In conditions 1 and 2, the derrick steel structure was simulated with single damage. Figures 10(a) and 10(b) show the significant mutations of the damage sensitivity index of sensors 6 and 7, which is consistent with the presupposition of damage position. Therefore, the single damage of the derrick steel structure can be accurately identified based on the HHT marginal spectrum amplitude curvature difference and IMF instantaneous energy curvature difference. Condition 3 simulates the double damage of the derrick steel structure. Figure 10(c) clearly shows that the damage-sensitive index of sensors 3 and 4 mutated and significantly changed. Sensors 9 and 10 had the largest amplitude of variation of the damagesensitive index with the adjacent value, which is also consistent with the preset double-damage position. Therefore, the double-damage position of the derrick steel structure can also be identified by the damage sensitivity index.

\section{Conclusions}

Vibration sensors have been widely studied and applied to collect vibration signals to detect the structural state of damage in health-monitoring technology. We only used the low-level vibration information of the derrick steel structure to propose a damage identification method for derrick steel structures based on the HHT marginal spectrum amplitude curvature difference.

(1) The HHT marginal spectrum amplitude curvature difference is related to the position of damaged elements. From the comparison with IMF instantaneous energy curvature difference, the position of damaged elements and the weak damage of the derrick steel structure can be accurately identified based on the HHT marginal spectrum amplitude curvature difference.

(2) The HHT marginal spectrum amplitude curvature difference can qualitatively analyze the damage degree of the elements, and the larger absolute value of the HHT marginal spectrum amplitude curvature difference between two ends of the damaged element increases with the increase in damage degree of the element.

(3) The simulation experiment of the damage location of the derrick steel structure shows that the damage identification method for derrick steel structures based on the HHT marginal spectrum amplitude curvature difference and IMF instantaneous energy curvature difference can extract the low-level vibration information. Thus, we can correctly identify the location of damaged elements and easily obtain 
the low-level vibration information of derrick steel structures in practice, which indicates that the method is reliable and easy to operate.

\section{Conflicts of Interest}

The authors declare that there are no conflicts of interest regarding the publication of this paper.

\section{Acknowledgments}

This work was supported by Hebei Provincial Natural Science Foundation of China (no. E2015203190) and Key Project of Natural Science Research in Colleges and Universities of Hebei Province (Grant no. ZD2015050).

\section{References}

[1] D. Han, Z. Li, and G. Zhou, "Simulation model for ultimate bearing capacity of in-service oil drilling derrick," Shiyou Xuebao/Acta Petrolei Sinica, vol. 28, no. 2, pp. 120-128, 2007.

[2] J. Liu, G. Zhou, and G. Han, "Simulation model for in-service rig derrick based on finite dynamic model," Shiyou Xuebao/Acta Petrolei Sinica, vol. 30, no. 5, pp. 788-792, 2009.

[3] D. D. Bueno, C. R. Marqui, V. Lopes Jr., M. J. Brennan, and D. J. Inman, "Structural damage identification and location using grammian matrices," Shock and Vibration, vol. 19, no. 3, pp. 287299, 2012.

[4] D.-Y. Han and P.-M. Shi, "Damage identification of derrick steel structures based on frequency and equivalent damage coefficient," Gongcheng Lixue/Engineering Mechanics, vol. 28, no. 9, pp. 109-114, 2011.

[5] H. Dongying and S. Peiming, "Identification of derrick steel structures damage based on frequency and bp neural network," China Safety Science Journal, vol. 18, no. 10, pp. 118-123, 2008.

[6] Z. Qu, S. Jin, H. Feng, Z. Zeng, Y. Zhou, and J. Li, "Multi-scale chaotic characteristic analysis of detection signals in pipeline pre-warning system based on empirical mode decomposition," Shiyou Xuebao/Acta Petrolei Sinica, vol. 29, no. 2, pp. 313-316, 2008.

[7] Y. Zhang, S. Jin, J. He, S. Chen, and J. Li, "Extraction method for pipeline leakage feature based on dynamic pressure signal," Shiyou Xuebao/Acta Petrolei Sinica, vol. 31, no. 2, pp. 338-342, 2010.

[8] J. Sun, S. Jin, and J. Sun, "Monitoring and pre-warning system for security of pipelines based on data fusion of multi-seismic sensors," Shiyou Xuebao/Acta Petrolei Sinica, vol. 30, no. 3, pp. 465-468, 2009.

[9] N. E. Huang, Z. Shen, S. R. Long et al., "The empirical mode decomposition and the Hilbert spectrum for nonlinear and non-stationary time series analysis," The Royal Society of London. Proceedings. Series A. Mathematical, Physical and Engineering Sciences, vol. 454, no. 1971, pp. 903-995, 1998.

[10] H. G. Chen, Y. J. Yan, and J. S. Jiang, "Vibration-based damage detection in composite wingbox structures by HHT," Mechanical Systems and Signal Processing, vol. 21, no. 1, pp. 307-321, 2007.

[11] H. Chen, Y. Yan, J. Jiang, and Q. Liu, "Application of HilbertHuang transform to damage detection for wing box section," Zhendong yu Chongji/Journal of Vibration and Shock, vol. 25, no. 3, pp. 81-98, 2006.
[12] H. G. Chen, Y. J. Yan, W. H. Chen, J. S. Jiang, L. Yu, and Z. Y. Wu, "Early damage detection in composite wingbox structures using hilbert-huang transform and genetic algorithm," Structural Health Monitoring, vol. 6, no. 4, pp. 281-297, 2007.

[13] D. Pines and L. Salvino, "Structural health monitoring using empirical mode decomposition and the Hilbert phase," Journal of Sound and Vibration, vol. 294, no. 1-2, pp. 97-124, 2006.

[14] H. Li, X. Deng, and H. Dai, "Structural damage detection using the combination method of EMD and wavelet analysis," Mechanical Systems and Signal Processing, vol. 21, no. 1, pp. 298306, 2007.

[15] N. Cheraghi and F. Taheri, "A damage index for structural health monitoring based on the empirical mode decomposition," Journal of Mechanics of Materials and Structures, vol. 2, no. 1, pp. 43-62, 2007.

[16] D. Rezaei and F. Taheri, "Experimental validation of a novel structural damage detection method based on empirical mode decomposition," Smart Materials and Structures, vol. 18, no. 4, Article ID 045004, 2009.

[17] D. Rezaei and F. Taheri, "Damage identification in beams using empirical mode decomposition," Structural Health Monitoring, vol. 10, no. 3, pp. 261-274, 2011.

[18] H. Chen, L. Zhang, J. Li, and W. Chen, "Improved EMDbased structural damage feature extraction," Zhendong Ceshi Yu Zhenduan/Journal of Vibration, Measurement and Diagnosis, vol. 32, no. 4, pp. 645-648, 2012.

[19] L. Cao, A. Li, Y. Deng, and Y. Ding, "Structural damage alarming based on empirical mode decomposition," Zhendong Ceshi Yu Zhenduan/Journal of Vibration, Measurement and Diagnosis, vol. 32, no. 3, pp. 392-396, 2012.

[20] Y.-C. Ren and P. Weng, "Structural damage detection based on improved Hilbert-Huang transform," Zhendong yu Chongji/Journal of Vibration and Shock, vol. 34, no. 18, pp. 195-199, 2015.

[21] X.-M. Wang, F.-L. Huang, G. Ma, and J.-J. Liu, "An HHTbased method to eliminate short-time strong disturbance from measured signals of bridge," Journal of Central South University of Technology (English Edition), vol. 14, no. 6, pp. 848-852, 2007.

[22] B. Li, C. Zhang, and Z. He, "HHT-based crack identification method for start-up rotor," Frontiers of Mechanical Engineering, vol. 7, no. 3, pp. 300-304, 2012. 


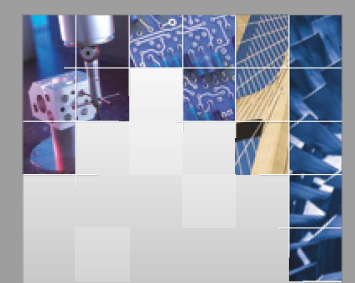

\section{Enfincering}
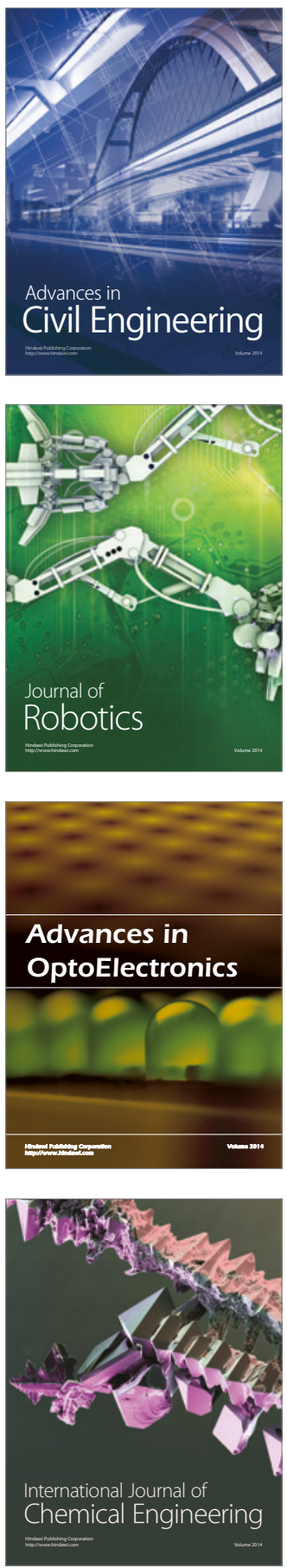

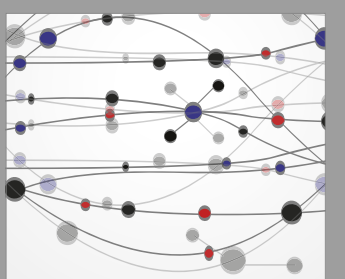

The Scientific World Journal

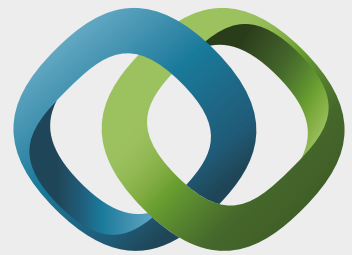

\section{Hindawi}

Submit your manuscripts at

https://www.hindawi.com
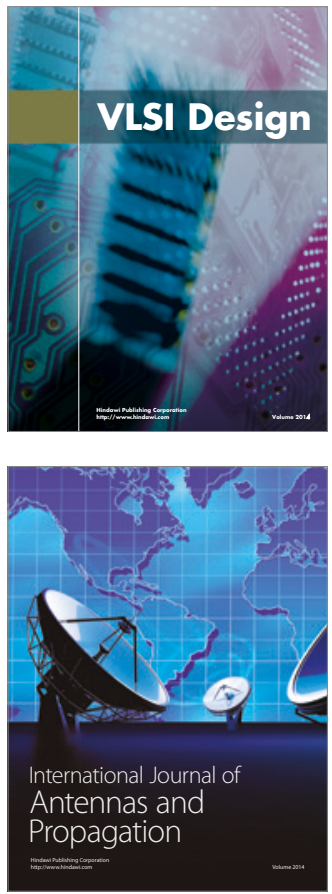

\section{Rotating}

Machinery
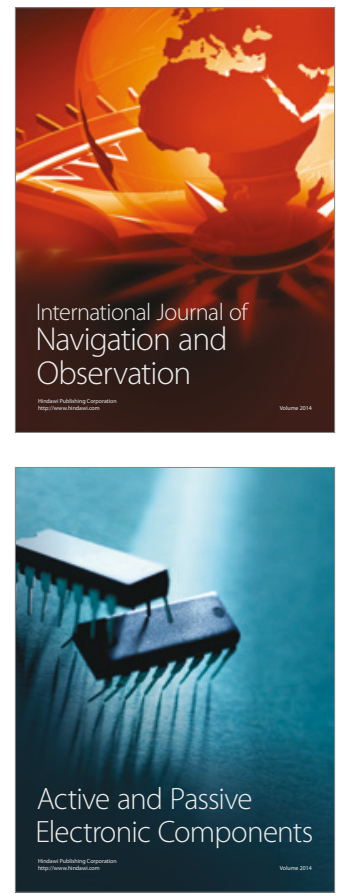
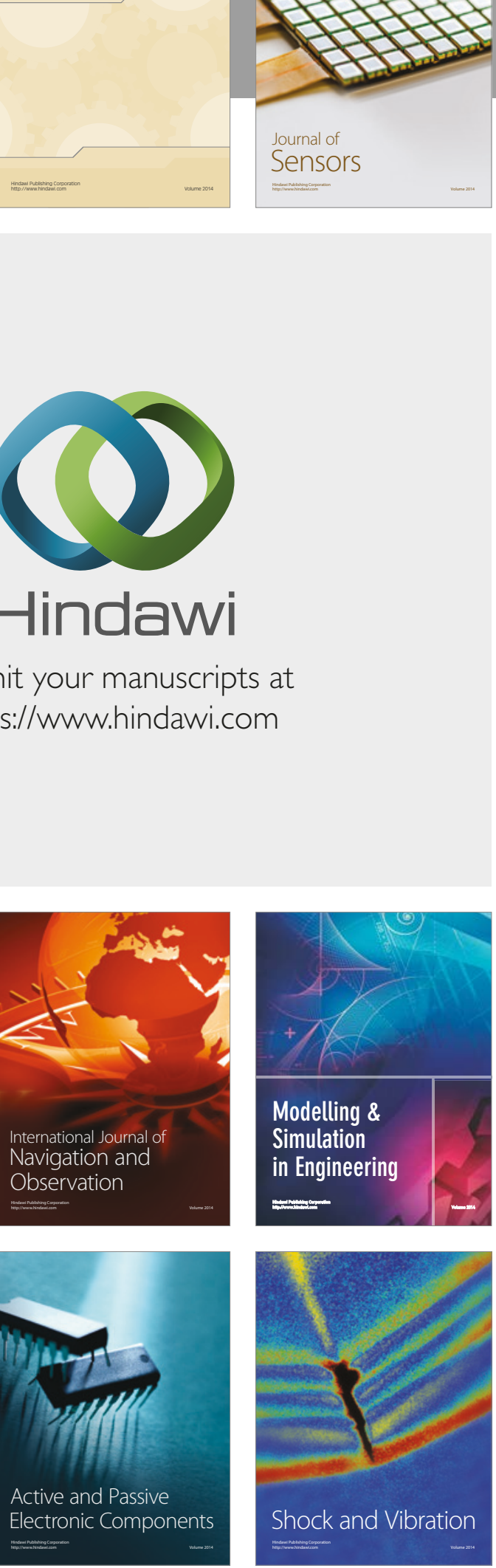
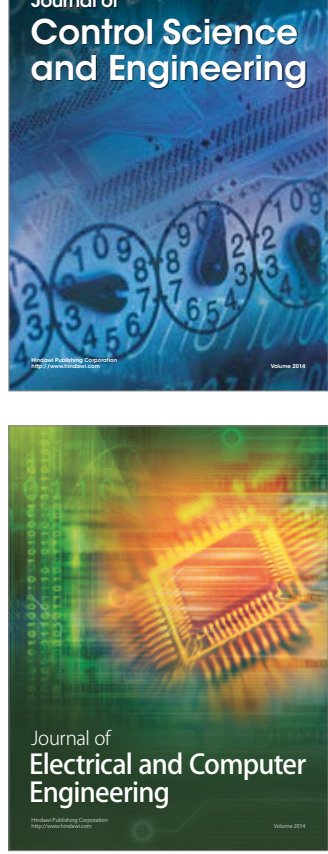

Distributed

Journal of

Control Science

and Engineering
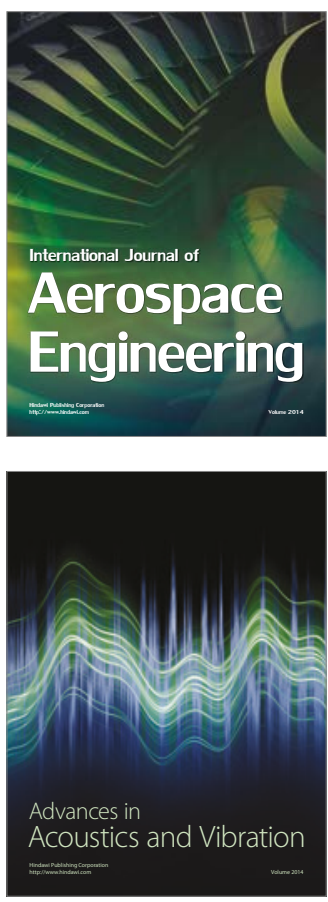

Sensor Networks 